\title{
Steady-State Two-Phase Flow in Porous Media: Laboratory Validation of Flow-Dependent Relative Permeability Scaling
}

\author{
Marios S. Valavanides1,*, Matthieu Mascle2, Souhail Youssef2 and Olga Vizika2
}

1Civil Engineering Department, University of West Attica, 12243 Attica, Greece

2IFP Energies Nouvelles, Rueil-Malmaison, France

\begin{abstract}
The phenomenology of steady-state two-phase flow in porous media is recorded in SCAL relative permeability diagrams. Conventionally, relative permeabilities are considered to be functions of saturation. Yet, this has been put into challenge by theoretical, numerical and laboratory studies that have revealed a significant dependency on the flow rates. These studies suggest that relative permeability models should include the functional dependence on flow intensities. Just recently a general form of dependence has been inferred, based on extensive simulations with the DeProF model for steady-state two-phase flows in pore networks. The simulations revealed a systematic dependence of the relative permeabilities on the local flow rate intensities that can be described analytically by a universal scaling functional form of the actual independent variables of the process, namely, the capillary number, $\mathrm{Ca}$, and the flow rate ratio, $r$. In this work, we present the preliminary results of a systematic laboratory study using a high throughput core-flood experimentation setup, whereby SCAL measurements have been taken on a sandstone core across different flow conditions -spanning 6 orders of magnitude on $\mathrm{Ca}$ and $r$. The scope is to provide a preliminary proof-of-concept, to assess the applicability of the model and validate its specificity. The proposed scaling opens new possibilities in improving SCAL protocols and other important applications, e.g. field scale simulators.
\end{abstract}

\section{Introduction}

The conventional use of saturation as the independent variable in two-phase flow in porous media (PM) is based on the oversimplifying assumption that disconnected fluidic elements of the non-wetting phase (NWP) (ganglia and droplets) do not move with the average flow but remain stranded in the pore medium matrix. This situation arises when flow conditions of 'relatively small values' of the capillary number are maintained. In those cases the disconnected NWP fluidic elements block part of the available flow cross-section by a fraction analogous to the average saturation and effect a relative reduction of the permeability of both the NWP and the wetting phase (WP). Nevertheless, there is ample experimental evidence that disconnected flow is a substantial and sometimes prevailing flow pattern [1-8].

Treating relative permeabilities as functions of the saturation is inefficient in providing a correct and specific-enough description of the process across the domain of all possible flow conditions. The issue is extensively discussed in [9]. A particular value in saturation does not necessarily imply that a unique disconnected structure (arrangement) of the NWP will settle-in. Disconnected structures of the NWP can be coarsely described by a spectrum of population density distributions, extending from distributions of 'many-andsmall' fluidic elements (droplets and small ganglia) to 'fewer-and-larger' fluidic elements (small and large ganglia). For any one of those cases, the corresponding superficial velocity of the disconnected NWP would not necessarily attain the same value. The latter would be the result of the 'negotiation' between the two factors inhibiting the transport of each phase, i.e. viscosity and capillarity, over the mass and momentum balances. Moreover, this 'negotiation' takes place within the 'regulatory framework' conformed by two critical 'stakeholders' or 'regulators', namely the particular structure of the PM and the externally imposed, flow conditions.

The term 'structure of the PM' comprises some appropriate, modeling ensemble of geometric/topologic characteristics of the pore network (dimensionality, genus, tortuosity, pore geometry etc.) as well as a set of physicochemical characteristics of the N/W/PM system, pertaining to- or associated with- wettability or to the favoring of interstitial, emulsion type flows. Wettability may be accounted by the values of the static contact angles of the N/W (NWP/WP) menisci against the pore walls. In that context, wettability conforms to the combination of the NWP, WP and PM surface characteristics and tripartite physicochemical affinities, as well as to hysteresis, expressed by the difference between the dynamic receding and advancing contact angles, pertaining to the motion of N/W menisci during drainage or imbibition of the WP within pores [10,14].

\footnotetext{
*Corresponding author: marval@uniwa.gr
} 
Flow conditions have an adverse effect on the momentum balance and, in particular, on the relevant magnitude of the Stokes flow resistances (due to bulk viscosity of the NWP and WP) and to Young-Laplace resistances (due to the contact forces between the N/W menisci and the pore walls). The former depend on local (micro /pore scale) velocity gradients (rates of deformations), in contrast to the latter that are relatively independent of rates of displacement of the menisci. From an energy point of view, the power dissipation due to bulk viscosity scales with the square of the local velocity gradients, whereas the capillarity-induced dissipation scales with the rates of displacement of the menisci. At relatively low values of the flow intensity (superficial velocity) viscosity effects are insignificant to capillarity effects; as flow intensity is progressively increased, viscosity takes over from capillarity and the flow progressively mutates from capillarity-dominated to transient capillary-viscosity- to viscosity-dominated characteristics [9].

As a consequence, for any two different cluster configurations (any two different population density distributions) of disconnected phase, the effective permeabilities of the WP and the NWP would differ and, therefore, the corresponding values of the relative permeability to the NWP would be different. Yet, those different cluster configurations (population distributions) and different values of the relative permeability could -in general- correspond to the same saturation value. In such situations, a universal, saturation-dependent description of the flow would be weak.

In addition, during core analysis, saturation is measured indirectly and cannot be externally imposed directly; it is only through control of a combination of pressure difference and/or flow rate of the NWP or WP that the system will attain an average saturation. If one wants to consistently and systematically describe the process in the entire flow regime (extending across extreme values in the capillary number and the viscosity ratio), one has to consider those variables that describe the externally imposed conditions and contain macroscopic kinematic information, e.g. the superficial velocity of each phase or, equivalently, the capillary number and the flow rate ratio. Moreover, saturation cannot adequately (or uniquely) describe the flow conditions. This is because saturation alone brings no definite input to the momentum balance, therefore it is questionable if it can provide any information on the kinetics of the macroscopic flow.

Observations of single phase flows within pore networks confirm that the macroscopic pressure gradient scales linearly with the superficial fluid velocity (Darcy's law). This seems to be a quite trustworthy modeling consideration in the case of two-phase flow as well, but only when very high superficial velocities are considered and capillary forces are negligible. However, at moderate/low velocities, when capillary forces are comparable to viscous forces, the macroscopic pressure gradient does not scale linearly with the flow rate. Experimental studies on steady-state two-phase flows in glass beads $[3,5,6]$, in glass bead columns [11], as well as in sand-pack columns [12], revealed that the non- linear relation between pressure gradient and flow rate can be described by generic power laws with different exponent values. The discrepancy between the values of the scaling exponents is attributed to differences associated with dimensionality of the pertinent variables, measurements pertaining to different flow conditions, dimensionality of the NWP/WP/PM system etc. Therefore, it is worth the effort to examine if these 'different' observations can be integrated in a universal power law relating appropriate, dimensionless variables of the process.

To this end, a first attempt in deriving a universal scaling functional form describing the flow dependency of relative permeability was recently proposed [13]. It is based on the results stemming from systematic, extensive simulations spanning 5 orders of magnitude in both the capillary number and flow rate ratio, implementing the DeProF model algorithm, build around a hybrid, true-to-mechanism, stochastic scale-up model for steady-state two-phase flows in pore networks [14].

The objective of the present work is three-fold:

(a) to examine the applicability of the proposed scaling on the basis of using typical R/SCAL measurements;

(b) to verify the existence, uniqueness and form of the locus of critical flow conditions, an inherent characteristic of such processes in terms of energy efficiency (see next section and [9]);

(c) to examine the potential of the proposed scaling and the underlying theoretical framework, in revealing the flow structure and/or network structure characteristics of any set of R/SCAL measurements (R/SCAL forensics).

To do so we have used a set of SCAL measurements on a typical sandstone core. Data were collected over a grid of specially selected flow conditions, spanning across up to 5 orders of magnitude on $C a$ and up to 10 orders on $r$. The laboratory study was performed independently, i.e. prior to the publication of the recently proposed flow-dependent relative permeability scaling; in that sense it can be characterized as a "blind study". With respect to (b), the existence of critical flow conditions (CFCs) has been already verified in an extensive review of published $\mathrm{R} / \mathrm{SCAL}$ relative permeability diagrams [15]; nevertheless, verification of the universal form of the locus of CFCs proposed in [9] was lacking a laboratory verification using the same core and across a broad domain of flow conditions ( $\mathrm{Ca}$ and $\mathrm{r}$ ).

The paper deploys in 3 sections. We start by providing the basic modeling assumptions for concurrent, immiscible, steady-state two-phase flows in porous media and present the basic structure of the proposed, flow-dependent relative permeability scaling. We continue with describing the SCAL experiments we have tapped-on (methods and materials). Then, we present the results of the laboratory examination and associated raw data calculations. Finally, we discuss "points of agreement/disagreement" between scaling predictions and actual measurements and we draw conclusions on the applicability of the proposed scaling or its potential exploitation in SCAL technology. 


\section{Basic Theoretical Background}

Consider the simultaneous, one-dimensional concurrent flow of a non-wetting phase (NWP, index $n$ ) and a wetting phase (WP, index w) across a porous medium control surface, $\tilde{A}$, at flow rates equal to $\tilde{q}_{n}$ and to $\tilde{q}_{w}$ respectively. (A tilde $\sim$ is used to indicate a dimensional variable, no tilde indicates a dimensionless variable.) The phenomenological fractional flow Darcy relations that describe the steady-state, fully developed process, whereby the pressure gradient, $(\Delta \tilde{p} / \Delta \tilde{z})$, is common in both fluids ([9], Appendix I), are given by eqn (1)

$$
\widetilde{U}_{i}=\frac{\tilde{q}_{i}}{\tilde{A}}=\frac{\tilde{k}}{\widetilde{\mu}_{i}} k_{r i}\left(\frac{\Delta \tilde{p}}{\Delta \tilde{z}}\right), \quad i=n, w
$$

In one-dimensional flow under steady-state conditions, especially when the flow is fully developed, the common pressure gradient in both phases can be expressed in reduced form as

$$
x=\frac{\Delta \tilde{p} / \Delta \tilde{z}}{(\Delta \tilde{p} / \Delta \tilde{z})^{1 \Phi}}=\frac{\Delta \tilde{p} / \Delta \tilde{z}}{\widetilde{\gamma}_{n w} C a / k}=\frac{\Delta \tilde{p}}{\Delta \tilde{z}} \frac{\tilde{\mu}}{\widetilde{\mu}_{w} \widetilde{U}_{w}}=\frac{1}{k_{r w}}
$$

where

$(\Delta \tilde{p} / \Delta \tilde{z})^{1 \Phi}$ is the pressure gradient corresponding to an equivalent saturated one-phase flow $(1 \Phi)$ of the WP at the same superficial velocity $\left(\widetilde{U}_{w}\right)$.

$\mathrm{Ca}$ is the capillary number, conventionally defined as

$$
C a=\tilde{\mu}_{w} \widetilde{U}_{w} / \tilde{\gamma}_{n w}
$$

$\tilde{\mu}_{w}$ is the viscosity of the WP and $\tilde{\gamma}_{n w}$ is the NWP/WP interfacial tension.

In eqn (2) the actual pressure gradient is divided by the pressure gradient of an equivalent one-phase flow of water at superficial velocity equal to $\widetilde{U}_{w}$ (the 2 nd component of the product). Note that, by definition, it is straightforward to verify that the reduced pressure gradient is essentially the inverse of the relative permeability of the WP.

The set of superficial velocities in eqn (1) may be appropriately reduced and replaced by a set of dimensionless variables, namely, the capillary number, $\mathrm{Ca}$, as defined previously in eqn (3), and the N/W flow rate ratio,

$$
r=\tilde{q}_{n} / \widetilde{q}_{w}=\widetilde{U}_{n} / \widetilde{U}_{w}
$$

At steady-state conditions in one-dimensional flow, the flow rate ratio, $r$, is equal to the mobility ratio, $r_{\lambda}$. The equivalence, expressed in the first part of eqn (5), is a conjecture resulting from direct flow analysis (see Appendix I in [9]).

$$
r=\frac{U_{n}}{U_{w}} \equiv \frac{1}{\kappa} \frac{k_{r n}}{k_{r w}}=r_{\lambda} \quad \Leftrightarrow \quad k_{r n}=\kappa r k_{r w}
$$

where

$$
\kappa=\tilde{\mu}_{n} / \tilde{\mu}_{w}
$$

is the NWP/WP bulk viscosity ratio.

Eqn (5) is very useful, as we may easily recover the $r_{i}$ value pertaining to any pair $i$ of measured $\left\{k_{r n}, k_{r w}\right\}_{i}$ values from a conventional (saturation dependent) relative permeability diagram. We may also recover a relative permeability by knowing the values of the other relative permeability and the flow rate ratio. Therefore, switching to the approach of flow-dependency may be readily applicable as all conventional relative permeability diagrams can still be used.

The conjecture that both fluids share a common pressure gradient, eqn (1), is also based on experimental evidence. In particular, eqn (5), i.e. the direct result of assuming a common pressure gradient, is verified by experiment. When measured values of flow rate ratio are plotted against calculated values of mobility ratio, they align on a straight line. Ample experimental evidence is provided in a recent work whereby $\sim 180$ relative permeability diagrams pertaining to steady-state conditions for a variety of N/W/PM systems have been reviewed [15]. The conjecture proves to be valid also in the present work, see the diagrams in Fig. 7. Because of the common pressure gradient [eqn (1)] and the equivalence between flow rate ratio and mobility ratio [eqn (5)], relative permeability curves intersect at a fixed value of the flow rate ratio, the so-called cross-over flow rate ratio value, $r x$. The latter is reciprocal to the viscosity ratio, $r_{x}=(1 / \kappa)$, as provided by eqn (5). This inherent characteristic of steady-state flows is universally observed in all relative permeability vs flow rate ratio diagrams ([9], [15] and Fig. 7).

In the line of the present work we will also analyze the results from an energy efficiency perspective. In this context, we will use the reduced (normalized) measure of the energy efficiency of the process, considered as the flow rate of the NWP per unit of total hydraulic power spent, or equivalently, provided externally to the N/W/PM system (say by the "pumps"), to maintain twophase flow (an essentially dissipative process) at any set of externally imposed flow conditions, $\mathrm{Ca}$ and $r$. The associated energy utilization factor, or energy efficiency index, $f_{E U}$, can be readily calculated in terms of macroscopic measurements [9], as

$$
f_{E U}=\frac{k_{r n}}{\kappa(r+1)}=\frac{r k_{r w}}{r+1}=\frac{r}{x(r+1)}
$$

For every fixed value of the capillary number, $\mathrm{Ca}$, there exists a single value of the flow rate ratio, $r^{*}$, for which the energy efficiency index, $f_{E U^{*}}$, attains a maximum value $[9,15]$. Moreover, for every N/W/PM system, a unique locus of energy efficiency maxima is formed, $r^{*}(C a)$. Flow conditions matching the $r^{*}(C a)$ locus are called critical flow conditions (CFCs). Critical flow conditions can be measured and identified by R/SCAL. The efficiency index provides a strong flow analysis and characterization tool [9].

Closing this passage, we need also to address the issue of selecting and using the flow rate ratio, $r$, instead of the fractional flow of the WP, $f_{w}$, as one of the two independent variables of the process - the other being the capillary number, $C a$. In the core analysis community the applicable standard is to refer to one of the fractional flow of the WP, $f_{w}$. Switching between $r$ and $f_{w}$ (or $f_{n}$ ) is readily provided through the 
transformation $f_{w}=r /(1+r)$. The use of the flow rate ratio, $r$, instead of the fractional flow, $f_{w}$, as independent variable, has the advantage of a more convenient description of the sought physical process, especially (or at least) around the critical flow conditions, whereby the identification of the critical flow rate ratio, $\log r^{*}(C a)$, becomes more evident and obvious. The implications in using $f_{w}$, or $f_{n}$, instead of $r$ are extensively discussed in [15]. As a general observation all energy efficiency diagrams $\left(\left\{r, f_{E U}\right\}_{i}\right)$ show a more uniformly smooth trend irrespective of the particular values of the system parameters and flow conditions examined in that review.

\subsection{Prediction of Steady-State Relative Permeabilities}

The mechanistic model DeProF for immiscible, steadystate two-phase flow in pore networks may be used to predict the reduced macroscopic pressure gradient, $x$, given the flow conditions and system properties. The model is based on the concept of decomposition in prototype flows, hence the acronym DeProF. It takes into account the pore-scale mechanisms and the sources of non-linearity caused by the motion of interfaces, as well as other complex, network-wide cooperative effects, to estimate the conductivity of each class of pore unit cells in a statistical sense. It implements effective medium theory with appropriate expressions for pore-tomacro scale consistency for NWP and WP mass transport, to derive an implicit algebraic relation invoking the macroscopic pressure gradient, the capillary number, $\mathrm{Ca}$, the flow rate ratio, $r$, the viscosity ratio, $\kappa$, the dynamic advancing and receding contact angles, $\left(\theta_{A}, \theta_{R}\right)$ (wettability), and a set of parameters that describe the geometrical, topological and structural characteristics of the pore network, $\mathbf{x} p m$ [14].

Using the DeProF model, one can obtain the solution to the problem of steady-state two-phase flow in porous media in the form of the following transfer function,

$x=x\left(C a, r ; \kappa, \theta_{\mathrm{A}}, \theta_{\mathrm{R}}, \boldsymbol{x}_{\boldsymbol{p} m}\right)$

Implementation of the DeProF model is possible when analytical expressions of the pore network geometry are plausible and general expressions for the cell conductivities can be calculated for all possible flow configurations. In general, this is not the case for real porous media, but only for a certain class of model pore networks with analytically tractable pore geometry. Yet, one can benefit by scanning entire domains of flow conditions at relatively short times and therefore get a systematic and consistent overview of the behavior of the flow across orders of magnitude on flow conditions. In that context, DeProF can be used as a virtual,large core SCAL simulator.

In a recent work, extensive simulations implementing the DeProF algorithm have been carried out to derive maps that describe the dependence of the flow structure on the independent flow variables, namely the capillary number, $C a$, and the flow rate ratio, $r$, or, equivalently, to the reduced, superficial velocities of NWP and WP [14]. The simulations span 5 orders of magnitude in $\mathrm{Ca}$ $(-9 \leq \log C a \leq-4)$ and $r \quad(-2 \leq \log r \leq 2)$ over sufficiently fine steps. Fluid systems with various viscosity ratios (9 in total), have been examined.

Indicative DeProF model predictions of the reduced pressure gradient, $x$, are presented in Fig. 1 for a typical $\mathrm{NWP} / \mathrm{WP} / \mathrm{PM}$ system with viscosity ratio $\kappa=1.5$. The diagrams furnish the projections of the $x_{i j}\left(C a_{i}, r_{j}\right)$ predicted values on many constant- $\log C a_{i}$ planes in (a) and constant- $\log r j$ planes in (b). In that context, markers are connected into iso- $\mathrm{Ca}$ and iso- $r$ groups.

Referring to Fig. 1(a), at the high-end of the $\log r$ domain, all curves pertaining to constant- $C a$ values tend to bundle and align asymptotically to the straight (dashed) line. The corresponding functional form, eqn (9), states that at sufficiently large $C a$ values the reduced pressure gradient becomes a linear function of the flow rate ratio, with the linearity constant equal to the viscosity ratio, $\kappa=1.5$.

$$
\text { As } r \rightarrow \infty, \quad \log x=\log \kappa+\log r \Leftrightarrow x=\kappa r
$$

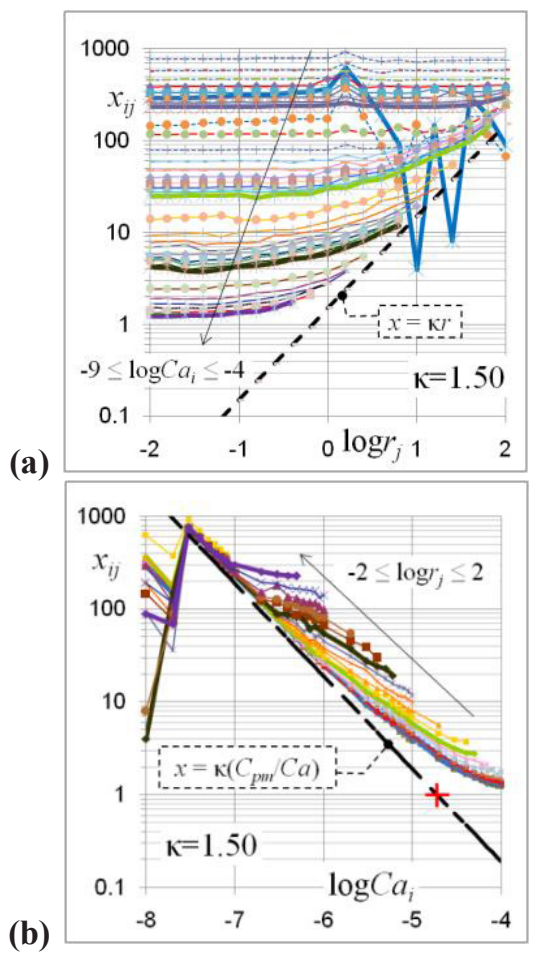

Fig. 1 Reduced pressure gradient values, $x$, for different values, (a) of the capillary number, $\mathrm{Ca}$, and (b) of the flow rate ratio, $r$. Both diagrams pertain to a typical value of the viscosity ratio, $\kappa=1.5$ function

We may observe a similar trend in Fig. 1(b). At the low-end of the $\mathrm{Ca}$ domain, all iso- $r$ curves tend to bundle and align asymptotically to a straight line, also illustrated with a dashed line. Now the functional form is given by the expression, up to a constant value, $C_{p m}$, associated to the N/W/PM system [13].

$$
\begin{aligned}
& \log C a \ll 0, \log x=\log \kappa-\log C_{p m}-\log C a \Leftrightarrow x= \\
& \kappa /\left(C_{p m} C a\right)
\end{aligned}
$$




\subsection{Universal Functional Form of Relative Permeabilities}

Based on these simulations it was possible to derive a universal function that can describe the dependence of the DeProF model predicted values $x_{i j}\left(\mathrm{Ca}_{i}, r_{j}\right)$ of the reduced pressure gradient for different values of the capillary number, $\mathrm{Ca}$, and the flow rate ratio, $r$, by the universal scaling form [13],

$x(\log C a, \log r)=A(\log C a)+\kappa r$

where the functional $A(\log C a)$ may be determined by any fitting procedure. The set of $(A i, C a i)$ values is plotted on the diagram of Fig. 2. The cross-markers are lined-up against two straight line asymptotes; one with negative inclination and the other coinciding with the horizontal axis; these meet at an oblate angle.

We may fit the set of $\left(\log C a_{i}, \log A_{i}\right)$ values by a function of the form,

$$
\log C a=\log C a_{0}+C_{0} /(\log A)^{2}-C_{3} \log A
$$

where $-1 / C_{3}$ is the gradient of the inclined asymptote, and $\log C a_{0}$ is the abscissa of the intersection of the two asymptotes and coefficient $C_{0}$ is a measure of the overall distance (approach) to the two asymptotes (the higher this value, the greater the distance of the apex of the swarm of markers to the asymptotes' intersection).

The coefficients values pertaining to the particular NWP/WP/PM model system (examined in the DeProF simulations) are: $C_{3}=1.1474, \log C a_{0}=-4.3$, and $C_{0}=0.003$. The thick red line is the curve $\log A(\log C a)$ that has been fitted to the raw data $\log A_{i}$ using eqn (12). Note, eqn (12) is an implicit function of $\log A$ in terms of $\log C a$. In particular, it is a cubic equation of $\log A$, which can be solved analytically to provide an explicit analytical expression $\log A\left(\log \mathrm{Ca} / \mathrm{Ca}_{0} ; C_{0}, C_{3}\right)$.

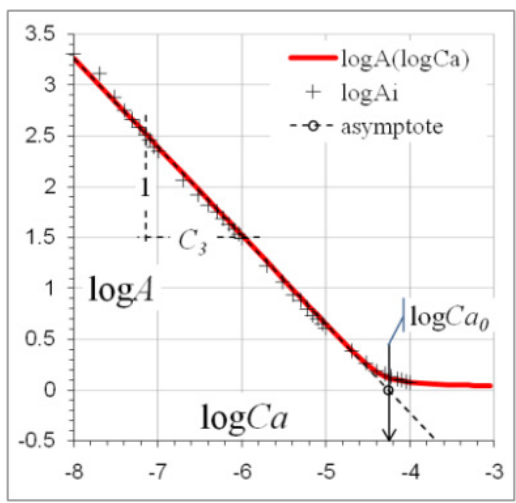

Fig. 2 Kernel function values $A$, in terms of $\mathrm{Ca}$. Cross-markers indicate values $\left(\log A_{i}, \log C a_{i}\right)$ of the kernel function used in eqn (11), calculated to fit the D eProF predicted data, Fig. 1(b). These have been fitted by the thick red line, using eqn (12). The black dashed line is an asymptote with gradient $\left(-1 / C_{3}\right)$.

Using the analytical expressions from eqs (11) and (12) with the aforementioned coefficient values, we may plot the reduced pressure gradient in terms of $\mathrm{Ca}$ and $r$, $x(\mathrm{Ca}, r)$. Comparing the diagrams of Fig. 2 to those of in Fig. 1, we observe that the DeProF predicted values $x_{i j}\left(\mathrm{Ca}_{i}, r_{j}\right)$ are recovered with great specificity, and that the trend at extreme flow conditions, maintains its physically-true characteristics (NWP flow is decoupled to the WP flow). Similar plots for other systems can be found in [13].

(a)
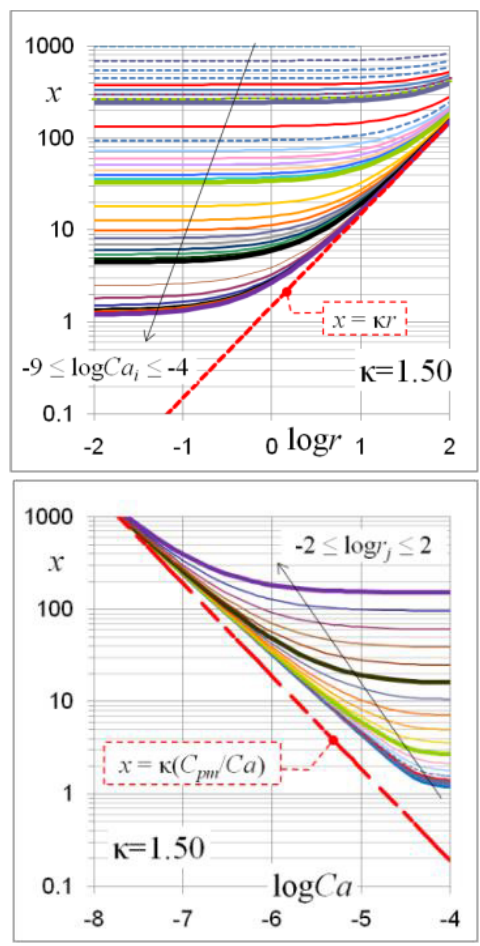

Fig. 3 Plots of the reduced pressure gradient values, $x$, in terms of the flow conditions, $\mathrm{Ca}$ and $r$. based on eqs (11) and (12); (a) iso- $\mathrm{Ca}$ curves; (b) iso- $r$ curves.

We also need to stress here that the particular form/shape of the kernel function $\log A(\log C a)$ produced in Fig. 2, as well as the form of eqn (12), come as a result of fitting the $\log A i$ values produced by the DeProF model simulations of a NWP/WP/PM system of intermediate wettability. Yet, the system we examined in the laboratory is water wet. To this end, it needs to be investigated if we may implement the same type of functional form for the kernel function [as eqn (12)] or we will have to derive a different one.

\section{Laboratory Materials and Methods}

A set of SCAL measurements was ran on a typical sandstone core, over a grid of specially selected flow conditions ( 88 in total), spanning across $\sim 6$ orders of magnitude on $C a$ and $\sim 6$ orders on $r$.

\subsection{Rock samples and fluids}

Sample used in this work is water-wet outcrop sandstone from a Clashach quarry, with porosity of $13 \%$ and mean permeability of $672 \mathrm{mD}$. Sample was cored with a diamond core drill with $1 \mathrm{~cm}$ internal diameter and cut to a length of $2 \mathrm{~cm}$. After being cored, the sample was dried in an oven for at least 48 hours at a temperature of $60{ }^{\circ} \mathrm{C}$. 
Two-phase flow measurements were conducted using synthetic dodecane oil and brine at $5 \mathrm{~g} / \mathrm{l} \mathrm{NaCl}$ (Sodium Chloride) for the low to intermediate capillary number. Viscosity at $20^{\circ} \mathrm{C}$ were respectively for oil and brine 1.5 $\mathrm{cp}$ and $1.07 \mathrm{cp}$. Interfacial tension was measured at $\tilde{\gamma}_{n w}=50 \mathrm{mN} / \mathrm{m}$. For intermediate to high capillary number, i.e. for $1 \mathrm{~g} / \mathrm{lt}$ of SDBS (sodium dodecylbenzene-sulfonate) was added to the brine to lower the interfacial tension to $1 \%$ of the original, i.e. to $\tilde{\gamma}_{n w}=$ $0.5 \mathrm{mN} / \mathrm{m}$. According to Graue et al [16], Clashach sandstone exhibits an Amott index of 0.45 which is typical of strongly water wet rock. During our experiments we have checked that the sample do not spontaneously imbibe oil.

\subsection{Experimental set-up and procedure}

Two phase flow measurements were conducted using the CAL-X set-up that has been recently designed for high throughput core-flood experimentation [17]. It is composed of an X-ray radiography facility, a fully instrumented multi-fluid injection platform and a dedicated X-ray transparent beryllium core holder. The local and averaged saturation $S_{w}$ is derived from the radiographs, captured every 10 seconds, using BeerLambert law for multi-material.

Steady State Method (SSM) was used to derive relative permeability. The SSM method involves the injection of both fluids at the inlet of the plug while measuring the differential pressure and the saturation within the core. Eqn (1) can be solved analytically if capillary pressure gradient is constant and end effects are

\begin{tabular}{|c|c|c|c|c|c|c|c|c|c|c|}
\hline Cycle & Type & $\begin{array}{l}\langle\mathrm{Nca}\rangle \\
{[\times 10.6]}\end{array}$ & \begin{tabular}{|c|}
$\left.\begin{array}{c}k \\
{[m d]}\end{array}\right]$ \\
\end{tabular} & WP & NWP & $\begin{array}{c}\text { IFT } \\
{[\mathrm{mN} / \mathrm{m}]}\end{array}$ & $\begin{array}{c}\mathrm{qn}_{\mathrm{n}} \\
{[\mathrm{cc} / \mathrm{min}]}\end{array}$ & $\begin{array}{c}q_{w} \\
{[\mathrm{cc} / \mathrm{min}]}\end{array}$ & $\begin{array}{l}\mathrm{S}_{\mathrm{w}} \\
{[-]}\end{array}$ & $\begin{array}{c}\Delta p \\
\text { [mbar] }\end{array}$ \\
\hline 01 & 1st Dr & 0.54 & \multirow{3}{*}{ ชิ } & \multirow{8}{*}{$\begin{array}{l}\bar{c} \\
\frac{\pi}{2} \\
\bar{c} \\
\text { c }\end{array}$} & \multirow{8}{*}{ 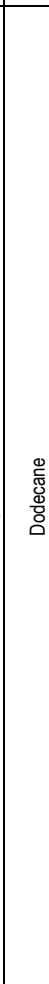 } & \multirow{8}{*}{50} & $\begin{array}{l}0.00001 \\
0.002 \\
0.048 \\
0.105\end{array}$ & $\begin{array}{c}0.097 \\
0.088 \\
0.044 \\
0.00001\end{array}$ & $\begin{array}{l}0.98 \\
0.67 \\
0.55 \\
0.28\end{array}$ & $\begin{array}{l}11 \\
64 \\
98 \\
80\end{array}$ \\
\hline 02 & 1st I & 0.54 & & & & & $\begin{array}{c}0.0934 \\
0.073 \\
0.048 \\
0.004 \\
0.00001 \\
\end{array}$ & $\begin{array}{c}.000001 \\
0.021 \\
0.045 \\
0.091 \\
0.097 \\
\end{array}$ & $\begin{array}{l}0.20 \\
0.28 \\
0.4 \\
0.49 \\
0.51 \\
0.52 \\
\end{array}$ & $\begin{array}{l}80 \\
124 \\
153 \\
199 \\
192 \\
\end{array}$ \\
\hline 03 & 2nd Dr & 0.56 & & & & & $\begin{array}{c}0.00001 \\
0.028 \\
0.046 \\
0.072 \\
0.092 \\
0.096\end{array}$ & $\begin{array}{c}0.097 \\
0.091 \\
0.046 \\
0.021 \\
0.004 \\
0.00001\end{array}$ & $\begin{array}{l}0.555 \\
0.545 \\
0.492 \\
0.452 \\
0.389 \\
0.27\end{array}$ & $\begin{array}{l}191 \\
189 \\
164 \\
130 \\
97 \\
82\end{array}$ \\
\hline 04 & $1 \mathrm{st} I \mathrm{~m}$ & 5.55 & \multirow{2}{*}{ ๙์ } & & & & $\begin{array}{c}1 \\
0.929 \\
0.736 \\
0.488 \\
0.239 \\
0.04 \\
0.00001\end{array}$ & $\begin{array}{c}.00001 \\
0.044 \\
0.246 \\
0.491 \\
0.736 \\
0.933 \\
0.974\end{array}$ & $\begin{array}{l}0.41 \\
0.19 \\
0.345 \\
0.42 \\
0.46 \\
0.48 \\
0.51 \\
0.53\end{array}$ & $\begin{array}{l}174 \\
407 \\
587 \\
670 \\
722 \\
682 \\
653\end{array}$ \\
\hline 05 & 2nd Dr & 5.58 & & & & & $\begin{array}{c}0.00001 \\
0.043 \\
0.246 \\
0.501 \\
0.746 \\
0.946 \\
0.99 \\
\end{array}$ & $\begin{array}{c}0.974 \\
0.933 \\
0.736 \\
0.49 \\
0.245 \\
0.046 \\
0.00001 \\
\end{array}$ & $\begin{array}{l}0.54 \\
0.52 \\
0.5 \\
0.473 \\
0.441 \\
0.384 \\
0.23 \\
\end{array}$ & $\begin{array}{l}650 \\
695 \\
713 \\
712 \\
629 \\
400 \\
180 \\
\end{array}$ \\
\hline 06 & 1st Dr & 5.60 & \multirow{3}{*}{ స్ర } & & & & $\begin{array}{c}0.00001 \\
0.046 \\
0.245 \\
0.502 \\
0.748 \\
0.944 \\
0.996 \\
\end{array}$ & $\begin{array}{c}.00001 \\
0.998 \\
0.932 \\
0.735 \\
0.491 \\
0.237 \\
0.046 \\
0.00001\end{array}$ & $\begin{array}{l}0.964 \\
0.75 \\
0.65 \\
0.574 \\
0.51 \\
0.415 \\
0.225 \\
\end{array}$ & $\begin{array}{l}97 \\
403 \\
561 \\
630 \\
612 \\
435 \\
243 \\
\end{array}$ \\
\hline 07 & 1st Im & 5.57 & & & & & $\begin{array}{l}0.996 \\
0.942 \\
0.743 \\
0.496 \\
0.232 \\
0.041 \\
0.00001\end{array}$ & $\begin{array}{c}0.000001 \\
0.045 \\
0.244 \\
0.49 \\
0.733 \\
0.93 \\
0.982 \\
\end{array}$ & $\begin{array}{l}0.2<5 \\
0.225 \\
0.39 \\
0.464 \\
0.505 \\
0.525 \\
0.55 \\
0.561 \\
\end{array}$ & $\begin{array}{l}240 \\
243 \\
430 \\
726 \\
871 \\
876 \\
828 \\
760 \\
\end{array}$ \\
\hline 08 & 2nd Dr/ & 5.56 & & & & & $\begin{array}{c}0.00001 \\
0.041 \\
0.236 \\
0.486 \\
0.742 \\
0.947 \\
0.994 \\
\end{array}$ & $\begin{array}{c}0.982 \\
0.932 \\
0.734 \\
0.49 \\
0.243 \\
0.043 \\
0.00001 \\
\end{array}$ & $\begin{array}{l}0.572 \\
0.561 \\
0.533 \\
0.504 \\
0.46 \\
0.387 \\
0.19 \\
\end{array}$ & $\begin{array}{l}760 \\
811 \\
945 \\
890 \\
817 \\
500 \\
245 \\
\end{array}$ \\
\hline
\end{tabular}

neglected. This condition can be assessed when fluids are injected at high flow rate. Yet, these assumptions as well as laminar flow (i.e Reynolds number less than 10) have to be confirmed. Laminar condition is verified since a linear relation between flow rate and pressure drop is respected in mono-phasic injection. To validate the constant capillary pressure gradient and negligible capillary end effect, we verify that the saturation at steady state condition is uniform along the sample, see example on Fig. 4.

The core has been systematically flooded, by successive cycles of drainage and imbibition at practically constant values of the nominal capillary number, $N \mathrm{Ca}$, defined over total flow and average viscosity as

$$
N_{C a}=\left(\widetilde{U}_{n}+\widetilde{U}_{w}\right)\left(\tilde{\mu}_{n}+\tilde{\mu}_{w}\right) /\left(2 \tilde{\gamma}_{n w}\right)
$$

The diagram in Fig. 5 illustrates a map of the broad range of scanned flow conditions - spanning across 5 orders of magnitude over $\mathrm{Ca}$ and 10 orders of magnitude over $r$. Markers connected with lines group successive cycles of drainage/imbibition, at nominal capillary number values, $\mathrm{NCa}_{\mathrm{Ca}}$, as indicated in the legend.

Note, $\mathrm{Ca}$ is defined by eqn (3) whereas $\mathrm{NCa}_{\mathrm{C}}$ is defined by eqn (13). Therefore,

$$
N_{C a} / C a=(1+r)(1+\kappa) / 2
$$

In total, 14 cycles of drainage and imbibitions have been run and 88 measurements have been taken. The source data measurements are presented in Table 1.

Table 1 The 88 source data measurements taken for each of the 14 cycles of drainage and imbibitions type injections of the core floods. The panel on the left is for high-IFT set-up, the

\begin{tabular}{|c|c|c|c|c|c|c|c|c|c|c|}
\hline Cycle & Type & $\begin{array}{l}\langle\mathrm{NCa}\rangle \\
{[\times 10.6]}\end{array}$ & $\left.\begin{array}{c}k \\
{[\mathrm{md}]}\end{array}\right]$ & WP & NWP & \begin{tabular}{|c|}
$\mathrm{IFT}$ \\
{$[\mathrm{mN} / \mathrm{m}]$}
\end{tabular} & $\begin{array}{c}\mathrm{q}_{\mathrm{n}} \\
{[\mathrm{cc} / \mathrm{min}]}\end{array}$ & $\begin{array}{c}\mathrm{q}_{\mathrm{w}} \\
{[\mathrm{cc} / \mathrm{min}]}\end{array}$ & $\begin{array}{l}\mathrm{S}_{\mathrm{w}} \\
{[-]}\end{array}$ & $\begin{array}{c}\Delta p \\
{[\mathrm{mbar}]}\end{array}$ \\
\hline 09 & 1st Dr & 55.17 & & \multirow{6}{*}{ 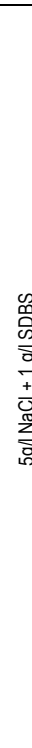 } & \multirow{6}{*}{$\begin{array}{l}8 \\
\text { वे } \\
\text { वे } \\
\text { c }\end{array}$} & \multirow{6}{*}{0.5} & $\begin{array}{c}0.00001 \\
0.0044 \\
0.048 \\
0.099\end{array}$ & $\begin{array}{c}0.98 \\
0.093 \\
0.047 \\
0.00001\end{array}$ & $\begin{array}{l}0.98 \\
0.58 \\
0.385 \\
0.14\end{array}$ & $\begin{array}{l}86 \\
58 \\
183 \\
40\end{array}$ \\
\hline 10 & 1st Im & 55.35 & & & & & $\begin{array}{l}0.099 \\
0.094 \\
0.074 \\
0.049 \\
0.005 \\
0.00001\end{array}$ & $\begin{array}{c}.00001 \\
0.004 \\
0.022 \\
0.048 \\
0.093 \\
0.098 \\
\end{array}$ & $\begin{array}{l}0.14 \\
0.15 \\
0.211 \\
0.311 \\
0.413 \\
0.431\end{array}$ & $\begin{array}{l}40 \\
576 \\
390 \\
180 \\
56 \\
28 \\
\end{array}$ \\
\hline 11 & 2nd Dr/ & 56.02 & & & & & $\begin{array}{c}0.00001 \\
0.005 \\
0.026 \\
0.05 \\
0.074 \\
0.096 \\
0.104 \\
\end{array}$ & $\begin{array}{c}0.098 \\
0.093 \\
0.073 \\
0.047 \\
0.022 \\
0.004 \\
0.00001 \\
\end{array}$ & $\begin{array}{l}0.43 \\
0.421 \\
0.41 \\
0.35 \\
0.25 \\
0.172 \\
0.165 \\
\end{array}$ & $\begin{array}{c}28 \\
32 \\
51 \\
105 \\
385 \\
746 \\
82 \\
\end{array}$ \\
\hline 12 & 1st Dr & 558.12 & & & & & $\begin{array}{c}0.00001 \\
0.041 \\
0.236 \\
0.493 \\
0.74 \\
0.94 \\
0.99\end{array}$ & $\begin{array}{c}0.0088 \\
0.937 \\
0.74 \\
0.494 \\
0.247 \\
0.048 \\
0.00001 \\
\end{array}$ & $\begin{array}{l}0.974 \\
0.9 \\
0.729 \\
0.47 \\
0.234 \\
0.143 \\
0.122\end{array}$ & $\begin{array}{c}86 \\
108 \\
230 \\
506 \\
1170 \\
1449 \\
250\end{array}$ \\
\hline 13 & 1st Im & 558.11 & న్ & & & & $\begin{array}{c}0.98 \\
0.937 \\
0.739 \\
0.494 \\
0.247 \\
0.048 \\
0.00001 \\
\end{array}$ & $\begin{array}{l}0.00001 \\
0.0439 \\
0.239 \\
0.493 \\
0.744 \\
0.935 \\
0.994 \\
\end{array}$ & $\begin{array}{l}0.122 \\
0.125 \\
0.191 \\
0.41 \\
0.624 \\
0.738 \\
0.783 \\
\end{array}$ & $\begin{array}{c}255 \\
1572 \\
1340 \\
520 \\
270 \\
134 \\
105 \\
\end{array}$ \\
\hline 14 & 2nd Dr & 558.69 & & & & & $\begin{array}{c}0.00001 \\
0.049 \\
0.236 \\
0.493 \\
0.74 \\
0.935 \\
0.99\end{array}$ & $\begin{array}{l}0.995 \\
0.935 \\
0.739 \\
0.494 \\
0.247 \\
0.048 \\
0.00001\end{array}$ & $\begin{array}{c}0.787 \\
0.746 \\
0.639 \\
0.437 \\
0.2 \\
0.111 \\
0.11\end{array}$ & $\begin{array}{l}105 \\
133 \\
267 \\
530 \\
1350 \\
1599 \\
280\end{array}$ \\
\hline
\end{tabular}
panel on the right for low-IFT set-up. 


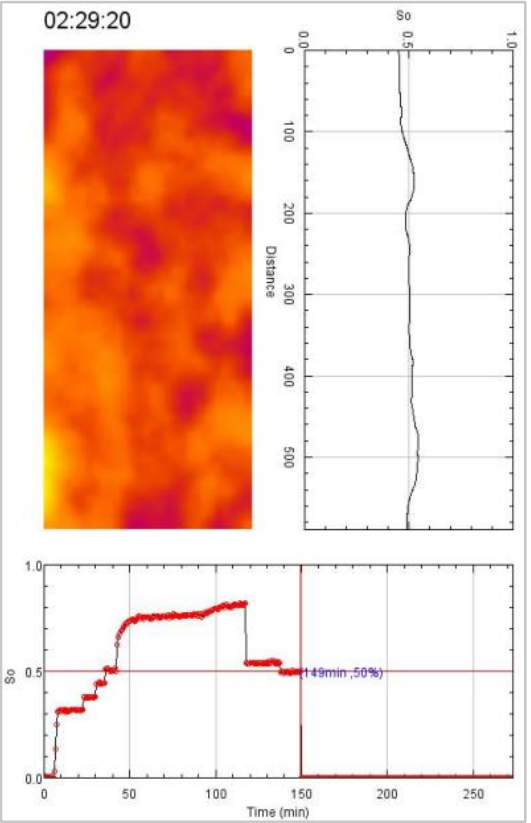

Fig. 4 Example of real-time saturation monitoring: 2D saturation map (upper left) and corresponding saturation profile (upper right); evolution of mean saturation as a function of time (low central)

\section{Results and discussion}

Pairs of NWP and WP relative permeability values $\left\{k_{r n}, k_{r w}\right\}_{i}$, were computed using eqn (1) for each measurement $i(i=1$, 88). These were then drawn into standard relative permeability diagrams in terms of the WP saturation, $S_{w i}$, for each one of the 14 different core flood cycles (or iso- $\mathrm{N}_{\mathrm{Ca}}$ groups). These diagrams are not presented here because of space limitation.

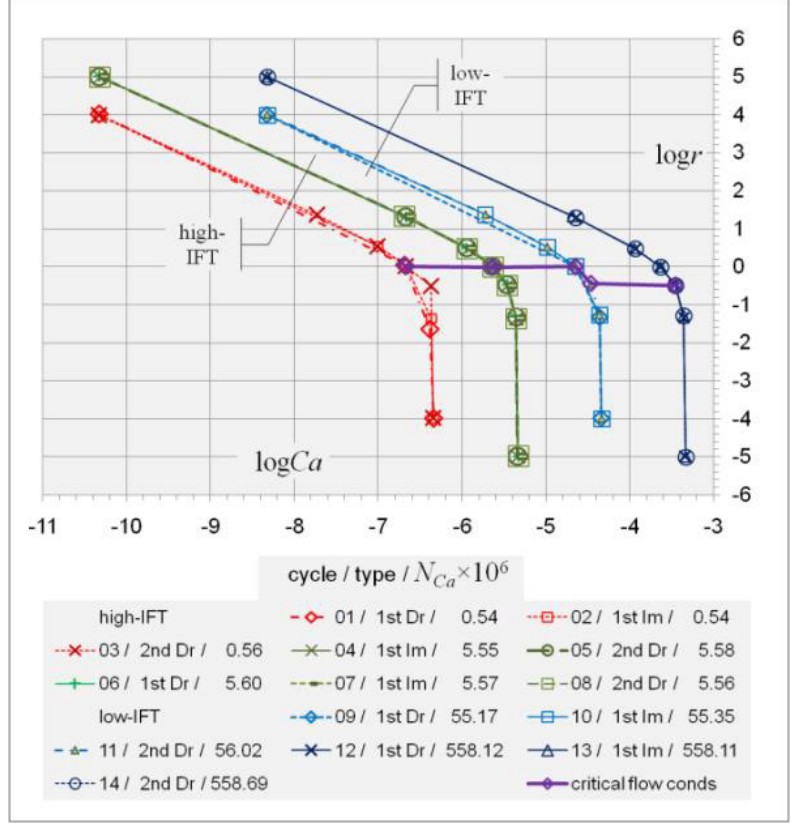

Fig. 5 Mapping of the 88 imposed flow conditions scanned during the 14 core-floods. Markers connected with lines are grouped into successive cycles of drainage/imbibitions, at constant values of the nominal capillary number, $\mathrm{NCa}$, as indicated in the legend.

For the record, they have been merged into a single diagram, see Fig. 6(a). No evidence of grouped correlations between $k_{r n}$, $k_{r w}$ and $S_{w}$ is observed (as expected). Yet, the picture becomes more clear when values of relative permeabilities, $\left\{k_{r n}, k_{r w}\right\}_{i}$, are plotted against corresponding values of the flow rate ratio, $r$. The diagram in Fig. 6 (b) is the result of merging the corresponding source diagrams in Fig. 7.

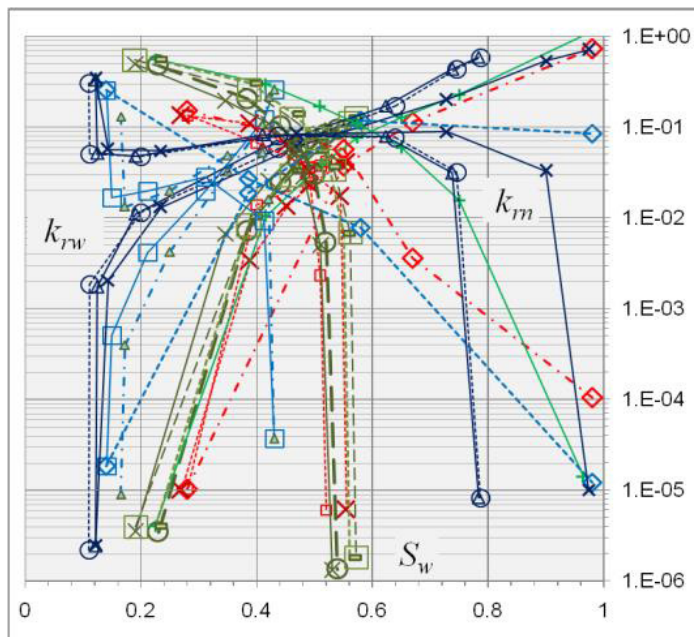

(a)

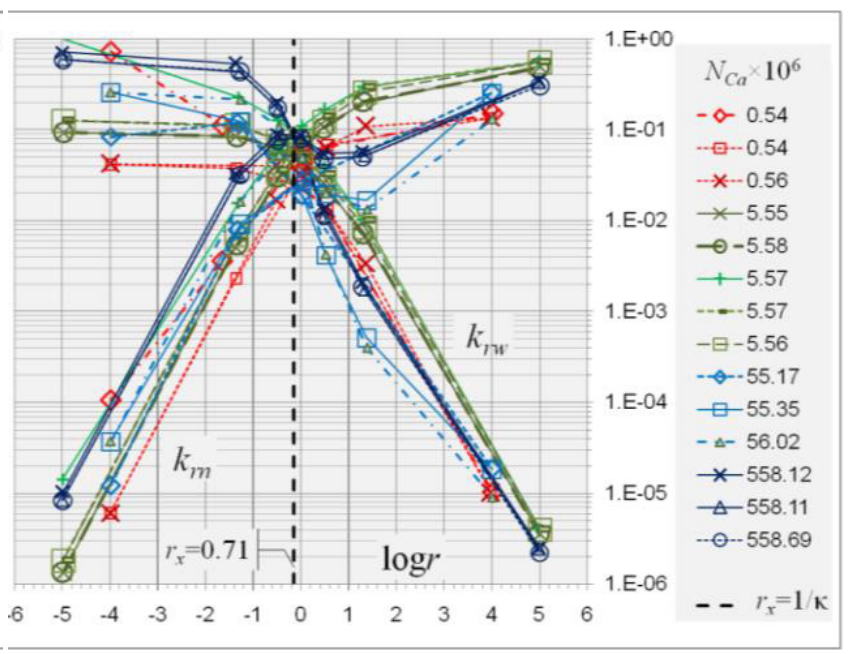

(b)

Fig. 6 Core-flood measured relative permeabilities, $k_{r n}, k_{r w}$, against corresponding mean values of WP saturation, $S_{w}$. (a) and flow rate ratio values, $\log r(\mathbf{b})$. In both diagrams, a total of 88 markers connected with lines are grouped into 14 successive cycles of drainage /imbibition, at constant values of the nominal capillary number, $N_{C a}$, as indicated in the legend. 

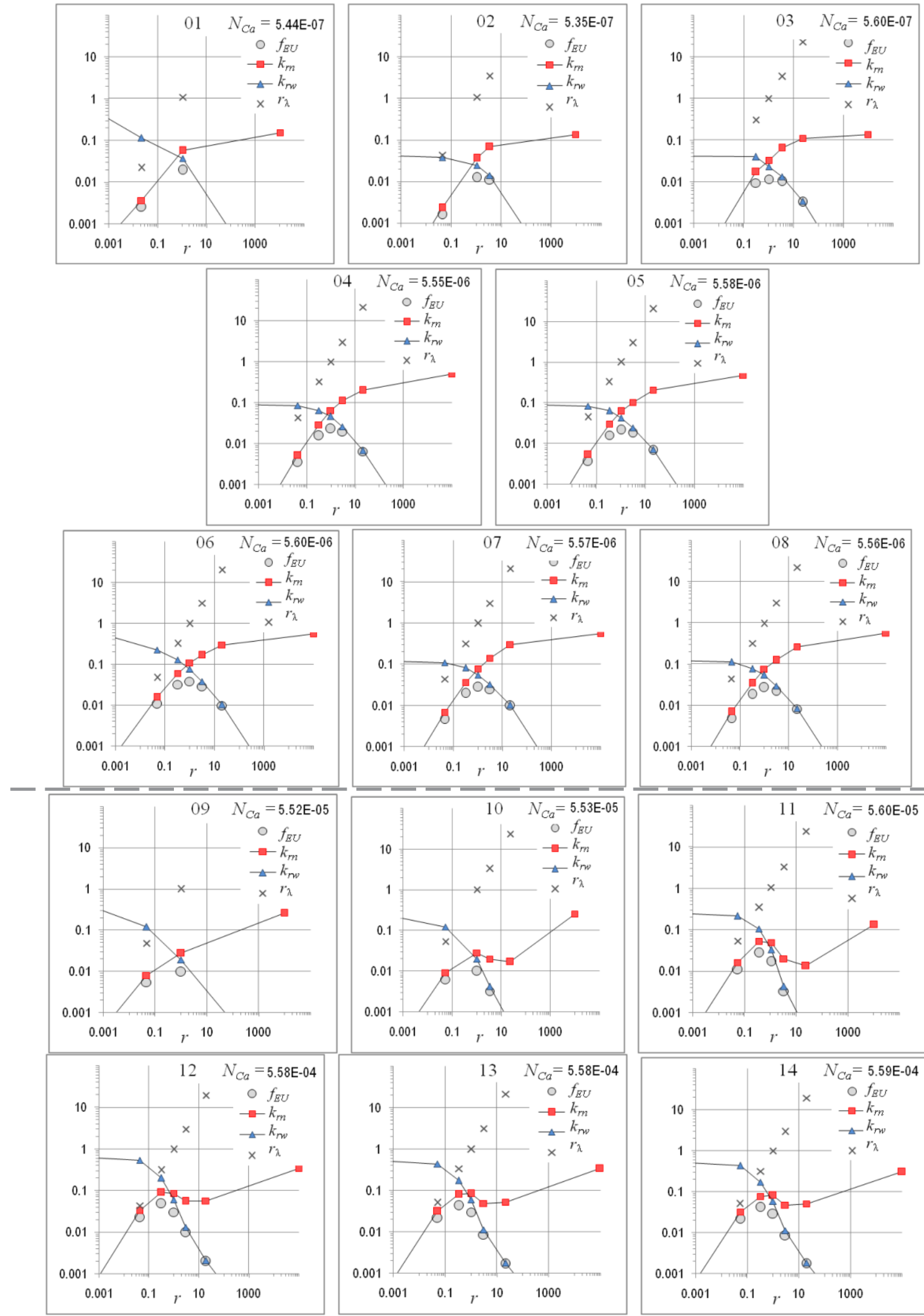

$N_{C a}=5.58 \mathrm{E}-06$ 


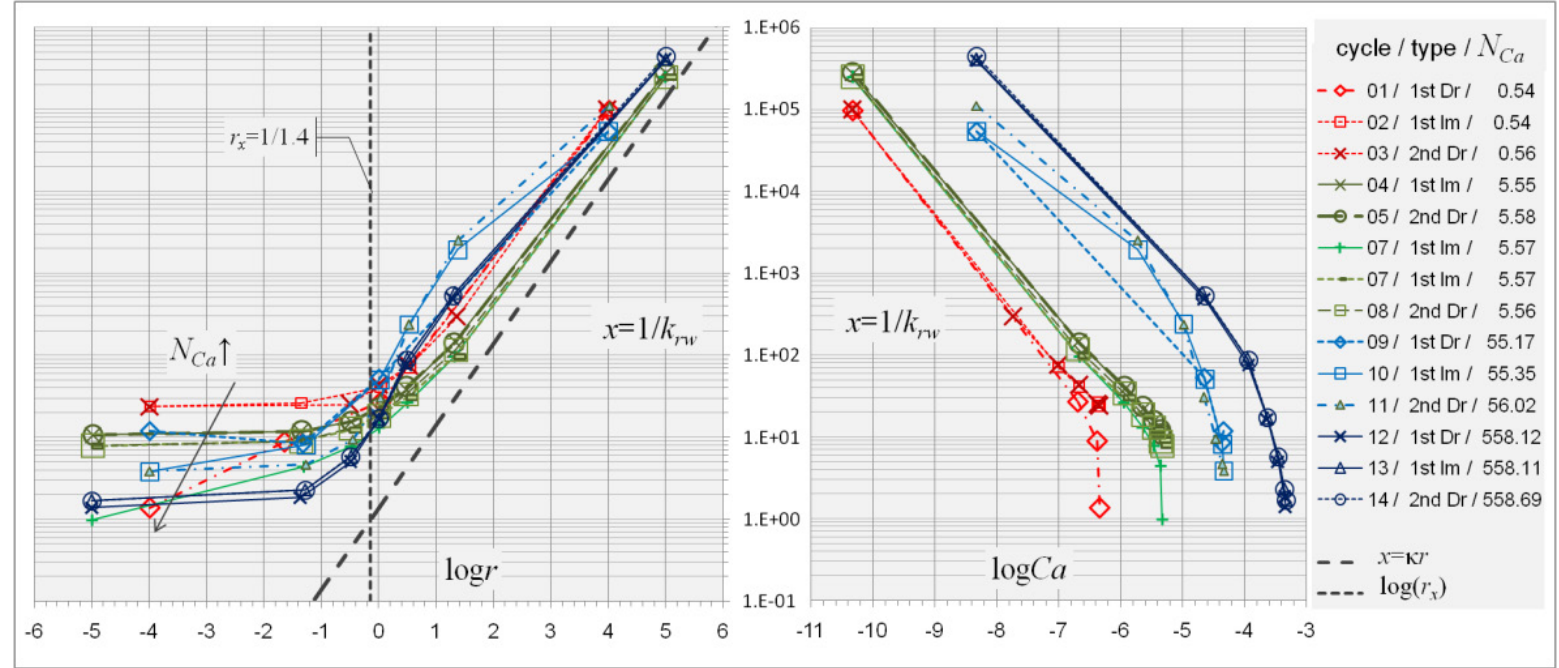

(a)

(b)

Fig. 8 Reduced pressure gradient values, $x$, measured during the core-floods. Data markers are organized in iso- $N_{C a}$ groups. (a) Dashed inclined line indicates the asymptotic line for flow decoupling provided by eqn (9). (b) Data group markers are clustered into two formations, each pertaining to a value of the interfacial tension (IFT $=50 \mathrm{mN} / \mathrm{m}$ (left cluster) and $0.5 \mathrm{mN} / \mathrm{m}$ (right cluster).

\section{Results and discussion}

Pairs of NWP and WP relative permeability values $\left\{k_{r n}, k_{r w}\right\}_{i}$, were computed using eqn (1) for each measurement $i(i=1,88)$. These were then drawn into standard relative permeability diagrams in terms of the WP saturation, $S_{w i}$, for each one of the 14 different core flood cycles (or iso- $N_{C a}$ groups). These diagrams are not presented here because of space limitation. For the record, they have been merged into a single diagram, see Fig. 6(a). No evidence of grouped correlations between $k_{r n}, k_{r w}$ and $S_{w}$ is observed (as expected).

Yet, the picture becomes more clear when values of relative permeabilities, $\left\{k_{r n}, k_{r w}\right\}_{i}$, are plotted against corresponding values of the flow rate ratio, $r$. The diagram in Fig. 6 (b) is the result of merging the corresponding source diagrams in Fig. 7.

Note, in the latter diagrams (Fig. 7), measured values of the actual flow rate ratio, $r$ [eqn (4)], and the calculated mobility ratio, $r_{\lambda}$ [eqn (5)], are aligned along a straight line with gradient 1 . Therefore, the conjecture of common pressure gradient in both fluids, as well as the transformation expressed in eqn (5), because of the underlying equivalence between $r$ and $r_{\lambda}$, are also verified. Note, in Fig. 6(b) and Fig. 7, all pairs of NW P and W P relative permeability curves intersect at the, socalled, cross-over flow rate ratio value, $r_{x}=(1 / \kappa)$, eqn (5). That value is indicated by the black short-dash line at $\log r_{x}=-\log (\kappa)$ in Fig. $6(b)$.

Reduced pressure gradient values, $x$, calculated as the reciprocal to the WP relative permeability, $k_{r w}$, [eqn (2)], are presented in Fig. 8. The diagram in Fig. 8 (a) presents data values in terms of $\log r$, classified into 14 iso- $N_{C a}$ groups. The general trend is similar to that provided by the proposed functional form, eqs (11) and (12), Fig. 1 and Fig. 3. Note, with increasing $r$, the asymptotic trend aligns to the dashed inclined line to a virtually decoupled flow state, as provided by eqn (9).
In Fig. 8 (b) reduced pressure gradient values, $x$, in terms of $\log C a$ are also presented in iso- $N_{C a}$ groups. The groups are clearly clustered into two formations (or "islands"). Each pertains to core floods performed with the same fluids but under different interfacial tensions (Section 3.1). The cluster on the left pertains to an interfacial tension (IFT) value $\tilde{\gamma}_{n w}=50 \mathrm{mN} / \mathrm{m}$, while the cluster on the right (higher $\mathrm{NCa}_{\mathrm{C}}$ ) to an IFT reduced by $1 \%, \tilde{\gamma}_{n w}=0.5 \mathrm{mN} / \mathrm{m}$. On the high- $\mathrm{Ca}$ region the trend is different when compared to that observed in the diagram of Fig. 1(b) [also Fig. 3 (b)]. Specifically, the mild and progressive drop of the $x$ values, as flow conditions shift to high- $C a$ regimes, contradicts the smooth but very steep (abrupt) drop of the pressure gradient measured in the core-floods (Fig. 8). Moreover, we may distinguish two similar behaviors. In the highIFT set-up (left cluster, groups 01-08) the drop in $x$ observed at increasing values of $\mathrm{Ca}$ is steeper when compared to the low-IFT set-up. In that same set-up (groups $09-11$ and 12-14) the drop in $x$ is progressive, yet its onset is practically independent of the $N_{C a}$. We will comment on this later on.

Using eqn (11), for every pair $\{x, r\}_{i}, i=1,88$ of the measured values, we may calculate a corresponding $A_{i}$ value for each flow condition imposed during the core-floods. The $A_{i}$ values are plotted in terms of $\log \mathrm{Ca}_{i}$ values on the diagram in Fig. 9. Comparing with Fig. 2, we observe the same differences in the trend (drop) of the $A_{i}$ values at high- $C a$ regimes. The trend in Fig. 9 is similar to that observed in Fig. 8(b). Note, also, data markers of the high-IFT set-up are aligned within a narrow band in the low- $C a$ regime, in contrast to the high-IFT set-up where markers are aligned to a wider band. The gradients of these bands in the low- $\mathrm{Ca}$ regime, could be considered as a "system identity" (compare with Fig. 2).

The overall trend indicates a drop of the effect of capillary forces at high- $\mathrm{Ca}$ regimes. The cause may probably be associated to interstitial, emulsion type flow, setting-in at high- $\mathrm{Ca}$ flow conditions. 
Fitting the $A_{i}$ data using the functional form expressed by eqn (12) does not succeed in capturing the trend at the high- $\mathrm{Ca}$ regime. An expression describing more consistently the trend at the high- $\mathrm{Ca}$ regime is

$$
\log A=A_{0}\left(1-\frac{\left|\log \left(C a / C a_{0}\right)\right|}{\left|\log \left(C a_{m} / C a_{0}\right)\right|}\right)^{\frac{1}{n}}-C_{g} \log \frac{C a}{C a_{0}}
$$

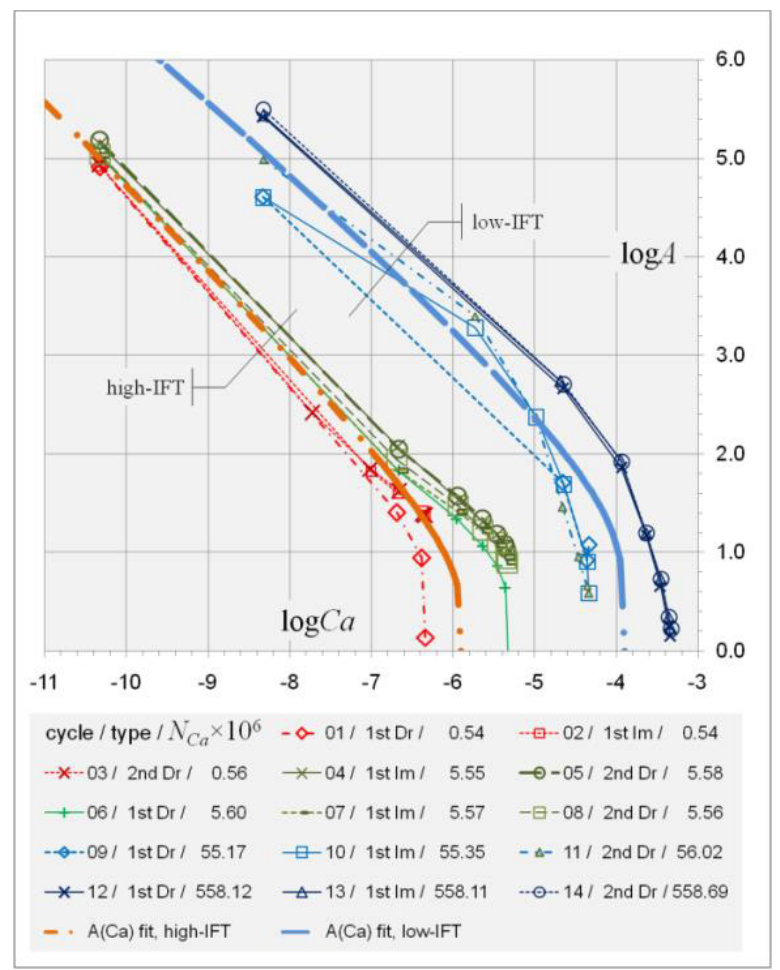

Fig. 9 The $\log A$ values in terms of $\log C a$, calculated from the core-flood data using eqn (11). The continuous orange dashdotted line fits high-IFT data, the blue dashed line fits low-IFT data, both using eqn (15).

The orange dash-dotted and the blue dashed lines fit the high-IFT and low-IFT data (Fig. 9). Coefficient values of the two fitting curves are presented in Table 2.

Table 2. Values of the $\log A$ fitting expressions coefficients

\begin{tabular}{|c|c|c|c|c|c|}
\hline IFT & $A_{0}$ & $\log C a_{m}$ & $\log C a_{0}$ & $C_{g}$ & $n$ \\
\hline High & 1.7 & -13 & -5.9 & 0.78 & 5 \\
\hline Low & 2.4 & -11 & -3.9 & 0.65 & 5 \\
\hline
\end{tabular}

We may now compare the dissimilar trends at high$\mathrm{Ca}$ regimes between the DeProF model predictions for a system of intermediate wettability, eqn (12), and the water wet system examined in the core-floods, eqn (15). To reveal any latent systematic trend and eventually get a better image of the interstitial flow structure, we have further investigated energy efficiency aspects of the core-floods within the particular N/W/PM system.

The diagram in Fig. 10(a) provides the map of the critical flow conditions (CFCs) identified from the core floods. The diagram is actually a blow-up of the central part of the diagram in Fig. 5 -focusing on the layout of the CFCs. The line connecting the CFCs attains a clearly formed S-shape, consistent to the $r^{*}(\mathrm{Ca})$ shape of the CFC locus on the universal energy efficiency map illustrated in [9] (cf. reference Figure 6 and 7). The dashed red horizontal line indicates the nominal value of the critical flow rate ratio for fully viscous flow conditions (as $\mathrm{Ca} \rightarrow \infty$ ). Provided the viscosity ratio value of the examined N/W system [9], this CFC nominal value is estimated to be $r_{\infty}^{*}=1 / \sqrt{\kappa}=0.845$ $\left(\log r_{\infty}^{*}=-0.073\right)$. The actual value attained within the lower part of the S-shape form, indicates an asymptotic trend $(C a \rightarrow \infty)$ towards $r_{\infty}^{*} \cong 0.32$. $\left(\log r_{\infty}^{*}=-0.496\right)$.

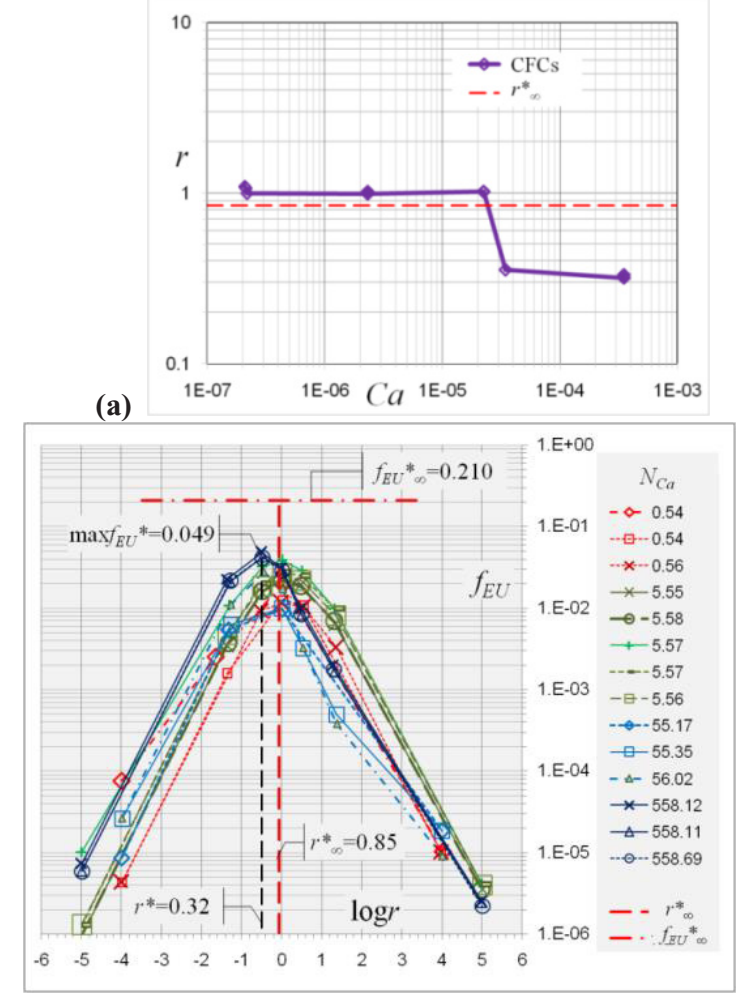

Fig. 10 Energy efficiency characteristics of the examined corefloods. (a) Detected critical flow conditions for various values of the capillary number, $r *\left(N_{C a i}\right)$, attaining an S-shape form. The diagram is a blow-up of the diagram in Fig. 5. (b) Energy efficiency values attained in terms of the flow rate ratio, $r$, grouped in iso- $\mathrm{NCa}$ core floods.

We may further examine the energy efficiency characteristics of the core floods, presented in the diagram of Fig. 10(b). The dash-dotted red horizontal line indicates the nominal value of the maximum attainable energy efficiency for the particular $\mathrm{N} / \mathrm{W}$ system, the "ceiling of efficiency", toping at $f_{E U_{\infty}}^{*}=$ $1 /(1+\sqrt{\kappa})^{2}=0.2096$ as $C a \rightarrow \infty$ (cf. eqn (30) in [9]), expected to be met at $r_{\infty}^{*}=1 / \sqrt{\kappa}=0.845\left(\log r_{\infty}^{*}=\right.$ $-0.073)$. Yet, the energy efficiency of the examined core floods reached an actual maximum value, $\max f_{E U}^{*}=-0.049$, at $r \cong 0.32(\log r=-0.496)$. It is obvious that the actual values of energy efficiency attained at large $C a$, are rather low when compared to the nominal, maximum values pertaining to the particular N/W system.

Overall, the observed differences are associated to high- $\mathrm{Ca}$ flow conditions. This strange behavior may be correlated to two factors favoring the setting of emulsion type flow: wettability (the examined system is $100 \%$ 
water wet) and emulsification, affecting the structure of the interstitial flow(s). The aforementioned nominal values $\left(r_{\infty}^{*}, f_{E U \infty}^{*}\right)$ are estimated considering the ideal case of minimization of capillarity effects at very large $C a$ values (see eqn (30) and Appendix II in [9]). In that modeling approach, capillarity effects are mainly attributed to the net Young-Laplace resistances at the contact lines, when menisci migrate downstream, by taking into account the hysteresis between the dynamic advancing and receding contact angles of menisci. If the flow intensities (superficial velocities) are large, power dissipation due to viscosity resistances prevails over power dissipation due to capillarity hysteresis -as the former depends quadratically on flow intensity, whereas the latter linearly. In that situation the flow is decoupled of capillarity effects and a nominal value of the flow rate ratio maximizing the energy efficiency can be estimated analytically (assuming a rather oversimplifying modeling hypothesis of negligible menisci resistances, [9]), as $r_{\infty}^{*}=1 / \sqrt{\kappa}$.

Nevertheless, in reality, e.g. during core floods in $\mathrm{R} / \mathrm{SCAL}$ measurements, the NWP may disconnect in tiny droplets. (This is also taken into account in the DeProF model simulations albeit the system is of intermediate wettability.) The onset of emulsification is not only due to the reduction of the interfacial tension between the NWP and the WP, but also due to the pore scale flow dynamics and the pore network characteristics [18]. In general, there are two classes of emulsion flows in porous media depending on the size of the drops compared to the capillary throat diameter. For emulsions with smaller drops, the extra-pressure drop does not vary with capillary number and it is a function of the viscosity ratio, dispersed phase concentration and drop size distribution. For emulsions with larger drops, these may partially block the capillaries, leading to higher extra pressure differences at low capillary numbers [1, 19]. Likewise, part of the NWP droplet population may pass through larger throats without contacting the pore walls. In that case a significant fraction of the NWP flows free of contact-line resistances. The associated energy cost is reduced, allowing for a shifting of the critical flow rate ratio to lower values in order to reduce also the power dissipation due to the bulk viscosity of the NWP [9].

Moreover, in water-wet systems, the hysteresis effects are pronounced due to the large differences occurring between receding and advancing dynamic contact angles that, in turn, burden the flow with substantial capillarity-induced resistances at the low- $\mathrm{Ca}$ regimes. Nevertheless, there is a cut-off limit in pore scale velocities above which lubricating-films are formed and capillarity induced resistances may drop significantly. This is expressed as the steep drop in reduced pressure gradient values pertaining to the examined core-floods systems.

\section{Conclusions}

We have performed a preliminary assessment/validation of the specificity and applicability of a new flow- dependent relative permeability scaling law that takes explicitly into account capillarity effects. Flow dependency is associated to the flow intensities of the NWP and WP or, equivalently to the capillary number of the WP, $C a$, and the NWP/WP flow rate ratio, $r$. The proposed scaling is build around a kernel function, $\log A(\log C a)$, that provides a "transitioning bridge" between the decoupled character of the flow at the two extreme flow regimes (low-Ca/low- $r$ to high- $\mathrm{Ca} /$ high- $r$ flows). The validation was based on a set of 14 corefloods of a typical water-wet sandstone and for a variety of flow conditions spanning across 5 orders of magnitude on $C a$ and 10 orders of magnitude on $r$.

The results suggest that it is possible to derive and implement universal, true-to-mechanism functions of flow-dependent relative permeabilities. The critical step here is to guess or data-fit an appropriate form of the kernel function, $\log A(\log C a)$. The latter is associated to the complex character of the NWP/WP/PM system rheology manifesting itself across the entire $\mathrm{Ca}$ domain.

The specificity /applicability of the proposed scaling is substantial and deserves further investigation, mainly towards understanding the universal structure of the kernel function $\log A(\log C a)$. Because of the structure of the scaling, eqn (11), the mode of decaying of $\log A$ at high- $C a$ regimes, whether expressed by eqn (12) or eqn (15), is equally applicable and depends on the physicochemical properties of the N/W/PM system, e.g. wettability. The critical property of the kernel function should be a monotonous decay at the high- $\mathrm{Ca}$ regime.

Implementing the proposed flow-dependency analysis it was possible to get a consistent and well structured view of the flow in the examined core-floods. The calculated values of reduced quantities (reduced pressure gradient, kernel function) show a consistent dependency on flow conditions and can be used to reveal the underlying flow mechanisms. In particular, considering the physicochemical properties of the two fluids, the results suggest that emulsion type flow settlesin above a threshold value of the capillary number. This threshold value depends on the flow conditions and the interfacial tension between the two fluids.

Reviewing the present work in terms of reaching its objectives, we may conclude:

(a) Implementation of the proposed scaling is possible using typical R/SCAL measurements; moreover it was possible to rely on superficial measurements only, i.e. flow rates of the two phases, pressure drop across the core length, IFT, absolute permeability.

(b) the existence, uniqueness and form of the locus of critical flow conditions, $r^{*}(\mathrm{Ca})$, was confirmed. The Sshape type, confined between two asymptotic lines, $r_{0}^{*}$ and $r_{\infty}^{*}$ as proposed in [9] is also confirmed. Differences between nominal (expected) and actual (measured) values, of $r_{\infty}^{*}$ and $f_{E U \infty}^{*}$, are justified on the basis of the induced emulsification of the flow occurring at very high $\mathrm{Ca}$ values. In order to reveal the S-shaping in greater detail, in follow-up studies the flow conditions should be finely scanned /tuned in proximity to the transition zone of the CFC locus. 
(c) By implementing the proposed scaling and considering the underlying mechanisms of the interstitial flows, it was possible to explain the flow behavior as described by the macroscopic measurements. That was possible by examining the structure of the WP relative permeability, $k_{r w}$, and of the kernel function $A$, without any need to examine the flow at the pore scale using sophisticated equipment. Therefore, the proposed scaling could potentially serve as an additional tool for quality assessment of measurements (R/SCAL forensics).

Based on the results of this preliminary assessment, it would make sense to consider designing and deploying a systematic laboratory to investigate and reveal the effects of wettability, pore network structure, core size, etc. on the form of $A(\log C a)$. The scope would be to improve R/SCAL protocols, and develop more robust and accurate macroscopic scale models.

The authors thank the reviewers for their constructive comments. MSV acknowledges partial support by the Research Council of Norway through its Centres of Excellence funding scheme, project number 262644 .

\section{References}

1. C.T. Tsakiroglou, D.G. A vraam, A.C. Payatakes, "Transient and steady-state relative permeabilities from two-phase flow experiments in planar pore networks", Adv. Wat. Res., vol. 30, pp. 1981-1992, 2007, doi:10.1016/j.advwatres.2007.04.002

2. V.R. Guillen et al., "Capillary-driven mobility control in macro emulsion flow in porous media", Int. J. Multiphase Flow, vol. 43, pp. 62-65, 2012, doi:10.1016/j.ijmultiphaseflow.2012.03.001

3. K.T. Tallakstad et al., "Steady-State Two-Phase Flow in Porous Media: Statistics and Transport Properties", Phys. Rev. Let., vol. 102, 074502, pp. 14, 2009, doi:10.1103/PhysRevLett.102.074502

4. A. Georgiadis et al., "Pore-scale micro-computedtomography imaging: Nonwetting-phase cluster-size distribution during drainage and imbibition", Phys. Rev. E, vol. 88, 033002, pp. 1-9, 2013, doi:10.1103/PhysRevE.88.033002

5. O. Aursjo et al., "Film flow dominated simultaneous flow of two viscous incompressible fluids through a porous medium", Frontiers in Physics, vol. 2, no. 63, pp. 1-9, 2014, doi:10.3389/fphy.2014.00063

6. S.S. Datta, T.S. Ramakrishnan, D.A. Weitz, "Mobilization of a trapped non-wetting fluid from a three-dimensional porous medium", Phys. Fluids, vol. 26, 2014, 022002, 2014, doi:10.1063/1.4866641

7. R. Oughanem et al., "A Multi-Scale Investigation of Pore Structure Impact on the Mobilization of Trapped Oil by Surfactant Injection" Transp. Porous Media vol. 109, pp. 673-692, 2015, doi:10.1007/s11242-015-0542-5

8. R.T. Armstrong, J.E. McClure, M.A. Berrill, M. Rücker, S. Schlüter, S. Berg, "Beyond Darcy's law: The role of phase topology and ganglion dynamics for two-fluid flow", Phys. Rev. E, vol. 94, 043113, 2016, doi: 10.1103/PhysRevE.94.043113

9. M.S. Valavanides, "Review of Steady-State TwoPhase Flow in Porous Media: Independent Variables, Universal Energy Efficiency Map, Critical Flow Conditions, Effective Characterization of Flow and Pore Network", Transp. in Porous Media, vol. 123, no. 1, pp. 42-99, 2018, doi:10.1007/s11242-018-1026-1

10. R.E. Hinkley, M.M. Dias, A.C. Payatakes, "On the motion of oil ganglia in porous media", PhysicoChemical Hydrodynamics, vol. 8, no.2, pp. 185-211, 1987

11. S. Sinha et al, "Effective Rheology of Two-Phase Flow in Three-Dimensional Porous Media: Experiment and Simulation", Transp. in Porous Media, vol. 119, no. 1, pp. 77-94, 2017, doi:10.1007/s11242-017-0874-4

12. C.D. Tsakiroglou et al., "Steady-state two-phase relative permeability functions of porous media: A revisit", Int. J. Multiphase. Flow, vol. 73, pp. 34-42, 2015, doi:10.1016/j.ijmultiphaseflow.2015.03.001

13. M.S. Valavanides, "Universal, Flow Dependnet Relative Permeability Scaling for Steady-State TwoPhase Flows in Porous Media", SCA2018-066, in Int. Symp. of the Society of Core Analysts, Trondheim, N orway, A ug. 27-30, 2018, pp.1-9

14. M.S. V alavanides, "Oil Fragmentation, Interfacial Surface Transport and Flow Structure Maps for Two-Phase Flow in Model Pore Networks. Predictions Based on Extensive, DeProF Model Simulations", Oil Gas Sci. Tech., vol. 73, no.6, pp. 1-36, 2018, doi:10.2516/ogst/2017033

15. M.S. Valavanides, E. Totaj, M. Tsokopoulos, "Energy efficiency characteristics in steady-state relative permeability diagrams of two-phase flow in porous media", J. Pet. Sci. Eng., vol. 147, pp. 181201, 2016, doi:10.1016/j.petrol.2016.04.039

16. A. Graue et al, "Imaging Fluid Saturation Development in Long-Core Flood Displacements", SPE 17438-PA, in Proc. SPE 58th California Regional Meeting, Long Beach, California, 1988, pp. 363-372, doi:org/10.2118/17438-PA

17. S. Youssef, M. Mascle, O. Vizika., "High Throughput Coreflood Experimentation as a Tool for EOR Project Design", SPE-190166-MS, in SPE Improved Oil Recovery Conf., Tulsa, Oklahoma, USA, 14-18 April, 2018

18. E. Unsal, M. B roens, R.T. Armstrong, "Pore Scale Dynamics of Microemulsion Formation", Langmuir, vol. 32, pp. 7096-7108, 2016, doi: 10.1021/acs.langmuir.6b00821

19. S. Cobos, M.S. Carvalho, V. Alvarado, "Flow of oil-water emulsions through a constricted capillary", Int. J. Multiphase Flow, vol. 35, pp. 507515, 2009, doi: 10.1016/j.ijmultiphaseflow.2009.02.018 